\title{
GRADIENT-BASED OPTIMIZATION OF SPACECRAFT AND AIRCRAFT THERMAL DESIGN
}

\author{
Laurynas MAČIULIS (D) ${ }^{1, *}$, Rimantas BELEVIČIUS (D) ${ }^{2}$ \\ ${ }^{1}$ Antanas Gustaitis Institute of Aviation, Vilnius Gediminas Technical University, Vilnius, Lithuania \\ ${ }^{2}$ Faculty of Fundamental Sciences, Vilnius Gediminas Technical University, Vilnius, Lithuania
}

Received 17 June 2020; accepted 25 June 2020

\begin{abstract}
Steady-case thermal analysis plays an important role in dimensioning thermal control systems for spacecrafts and aircrafts. Usually a trial and error approach is used based on engineering judgement and experience. When thermal models become complex or there are conflicting thermal requirements, however, it becomes harder for an engineer to gain insight as to which design decisions will lead to better results. Numerical optimization, on the other hand, could provide a more robust approach for the thermal design of complex spacecraft or aircraft models. In this paper, we suggest a gradientbased multidisciplinary optimization of thermal models where the coupled derivatives of the multidisciplinary system are obtained with the adjoint method. We show that in the case of steady-state thermal analysis, there is an analytic solution of a partial derivatives of implicit heat-transfer equation that can be used to derive total derivatives of the system. We present a practical application of this method by solving a small interplanetary spacecraft thermal optimization problem consisting of one objective, 15 design variables, and 10 constraints. We found that by using gradient-based optimization with exact derivatives, the best results can be achieved by exploring the design space at multiple initial starting points without major computational overhead.
\end{abstract}

Keywords: thermal, aircraft, optimization, spacecraft, gradient, multidisciplinary, adjoint, heat-transfer, openMDAO.

\section{Introduction}

The spacecraft and aircraft thermal-control system is responsible for maintaining and controlling components' temperature within the required operating conditions. Numerical thermal analysis is one of the key methods in the design and performance assessment of such thermalcontrol systems. Although the selection of a viable thermal design approach is based on engineering judgement, detailed thermal analyses are always required to verify and refine the design. In an industrial environment, a set of commercial thermal analysis computer programs are typically used to accurately model the thermal environment by either finite difference or finite element methods. Depending on space mission or aircraft design requirements, the underlying thermal models can become quite complex, with thousands of thermal nodes and a multitude of design points at varying boundary conditions. With the expansion of model complexity, it becomes harder for an engineer to gain insight as to which design decisions will lead to better results. In industrial practice, this is mostly done by human trial and error, however, the use of numerical optimization methods could provide a more robust approach for thermal design.

This is especially relevant for passive thermal-control system design for small spacecrafts, such as CubeSats, which typically rely solely on the proper selection of conductive and radiative material properties. Thermal-control challenges in aviation usually arise in hypersonic and electric aircraft design, especially concerning aircraft engine cooling systems and heat exchangers. Electric aircraft pose a unique thermal design challenge and opportunity for optimization in that they lack a simple way to reject waste heat from the power train. While conventional aircraft reject most of their excess heat in the exhaust stream, this is not an option for electric aircraft (Falck et al., 2017). Indeed, the need for integrated thermal, power, and fuel management systems modeling for the design of modern composite aircraft has been highlighted in the literature (Bodie et al., 2010).

Despite the potential advantages offered by numerical optimization of spacecraft and aircraft thermal design,

*Corresponding author. E-mail: laurynas.maciulis@vgtu.lt 
there is a lack of research on this subject in the scientific literature. Some researchers have successfully solved the inverse design of the spacecraft thermal-control system by using a meta-heuristic generalized extremal optimization algorithm. The design solutions found were nonintuitive ones that were unlikely to occur as possibilities for a human designer (Galski et al., 2007). Others have suggested methodologies based on deterministic optimization algorithms that use finite difference approximation of the objective function gradient (Chari, 2009; Stout et al., 2013). Escobar et al. (2016) used genetic algorithms to automate the design of a satellite passive thermal control system, while Wang et al. (2002) investigated an aircraft engine cooling problem combined with a finite element solution of heat conduction using an optimization technique based on the Adaptive Response Surface Method.

Global optimizers based on evolutionary algorithms offer an advantage as they do not rely on gradients and are therefore easy to implement with black box models. Without gradients, however, algorithms must rely on sampling the design space at a cost that grows exponentially with the number of design variables, and in practice, this becomes prohibitive when there are more than 10 design variables (Hwang et al., 2013). The same problem is faced in response surface methods, otherwise known as surrogate models, which rely on sampling the design space of often expensive analysis codes (Forrester et al., 2008). Furthermore, global optimizers have difficulty handling constraints, whereas thermal design problems are dominated by temperature constraints. Similar disadvantages are inherent in finite-difference-based gradient optimizers. In their simplest form, these methods require repetition of the system analysis for every perturbed design variable, meaning it may be prohibitively costly, particularly if the system analysis is nonlinear and/or iterative (Sobieszczanski-Sobieski, 1990). Finite-difference approximation of the gradient is also prone to inaccuracy due to computational noise and truncation errors that may lead to convergence issues.

Previous research has also considered thermal design to be a single discipline problem, but in most cases it is multidisciplinary in the context of other sub-systems. We suggest a gradient-based method that uses exact analytic derivatives of the underlying thermal analysis model and accounts for coupling with the electrical power supply and attitude sub-systems of a spacecraft. In this paper, we focus on spacecraft thermal design, but the same principles could also be applied to aircraft.

\section{Principles of spacecraft thermal analysis and design}

The objective of all thermal-analysis codes is the solution of the general heat-transfer equation. One way to determine the solution is to approximate the physical satellite model using a finite-difference model. The nodes or sub-volumes are assumed to be isothermal, and physi- cal properties are assumed to be constant within a node. Some heat-transfer books refer to finite-difference-node meshes as lumped-parameter representations (Gilmore, 2002). The energy equation for each node $i$ can be written as (Meseguer et al., 2012):

$$
\begin{aligned}
& C_{i} \frac{\partial T}{\partial t}=Q_{\text {sun }, i}+Q_{\text {alb }, i}+Q_{\text {planet }, i}+Q_{\text {dis }, i}+ \\
& \sum_{j=1}^{n} G L_{i j}\left(T_{j}-T_{i}\right)+\sum_{j=1}^{n} G R_{i j}\left(T_{j}^{4}-T_{i}^{4}\right),
\end{aligned}
$$

where $T$ is temperature in Kelvin scale; $t$ is time; $Q_{\text {sun, }, i}, Q_{\text {alb }, i}, Q_{\text {planet }, i}$ are external Sun, albedo, and planetary infrared heat fluxes, respectively; $Q_{d i s, i}$ is the internally dissipated power; and $G L_{i j}$ and $G R_{i j}$ are the conductive and radiative coupling links between nodes $i$ an $j$, respectively. When all nodes in the model are considered, one obtains a system of ordinary differential equations that can be solved to give the time evolution of the temperature of each node. For thermal-control system dimensioning, however, steady-state calculations for the worst-case scenarios are typically performed (Meseguer et al., 2012). This is done by setting the left-hand side of equation (1) to zero, thus obtaining a system of algebraic nonlinear equations. This system of equations can be reduced to the matrix form:

$$
\boldsymbol{G L} \cdot\{T\}+\boldsymbol{G R} \cdot\{T\}^{4}+\{Q\}=0,
$$

where $T$ is a column vector of node temperatures, $Q$ is a column vector of node external heat fluxes, $\boldsymbol{G L}$ is a linear conductor matrix, and $\mathbf{G R}$ is a radiative conductor matrix. Each element in linear conductor matrix is given by:

$$
G L_{i j}=\frac{k A}{L},
$$

where $k$ is the thermal conductivity, $A$ is the cross-sectional area through which the heat flows, and $L$ is the distance between two adjacent nodes $i$ and $j$. Similarly, the radiative conductor elements are given by:

$$
G R_{i j}=\sigma \cdot \epsilon_{i} \cdot \alpha_{j} \cdot A_{i} \cdot F_{i j},
$$

where $\sigma$ is the Stefan-Boltzmann constant, $\epsilon$ is infrared emissivity, $\alpha$ is infrared absorptivity, $A$ is node area, and $F_{i j}$ is the geometric view factor. The geometric view factor shows which fraction of the heat radiated by the surface $i$ is directly incident on a surface $j$ (Gilmore, 2002).

We will refer to equation (2) as the direct problem as it is used to solve node temperatures by knowing the elements of GL, GR, and $Q$. The thermal engineer, on the other hand, is faced with an inverse problem:

$$
\begin{aligned}
\text { Find } & X \\
\text { Subject to } & T_{i, l b}<T_{i}<T_{i, u b} \\
& x_{i, l b}<x_{i}<x_{i, u b},
\end{aligned}
$$

where $X$ is the vector of design variables belonging to the feasible domain specified within the upper and lower bounds denoted by $x_{\mathrm{ub}}$ and $x_{\mathrm{lb}}$, respectively, while $T_{\mathrm{ub}}$ and $T_{\mathrm{lb}}$ define the upper and lower temperature bounds defined by thermal requirements, respectively. Any 
parameter that influences the residual value of equation

(2) can be considered as a design variable.

The overall process of solving the inverse problem (as shown in Figure 1) is based on trial and error as well as engineering judgement. It is further complicated by the fact that multiple design points need to be considered according to the specific space-mission scenario. High fidelity thermal models coupled with varying boundary conditions result in very large design spaces that could reach thousands of design variables (Hwang et al., 2013). Numerical optimization could enable a more robust approach to solving the inverse problem and provide the designer with more insight into where a better solution lies.

\section{Formulation of the multidisciplinary thermal optimization problem}

Instead of looking into thermal design as a single-discipline problem, we suggest formulating it within a multidisciplinary context. By looking into equation (1), we can see that it contains inputs from external heat fluxes and heat dissipation from equipment, meaning that the thermal problem is inherently coupled with the spacecraft attitude and power disciplines. This formulation would also make it easy to integrate the thermal discipline into a multidisciplinary optimization framework of the whole satellite as a system. Following the mathematical notation suggested by Martins and Lambe (2013), we formulate a thermal problem as a multidisciplinary feasible (MDF) optimization architecture in the form of:

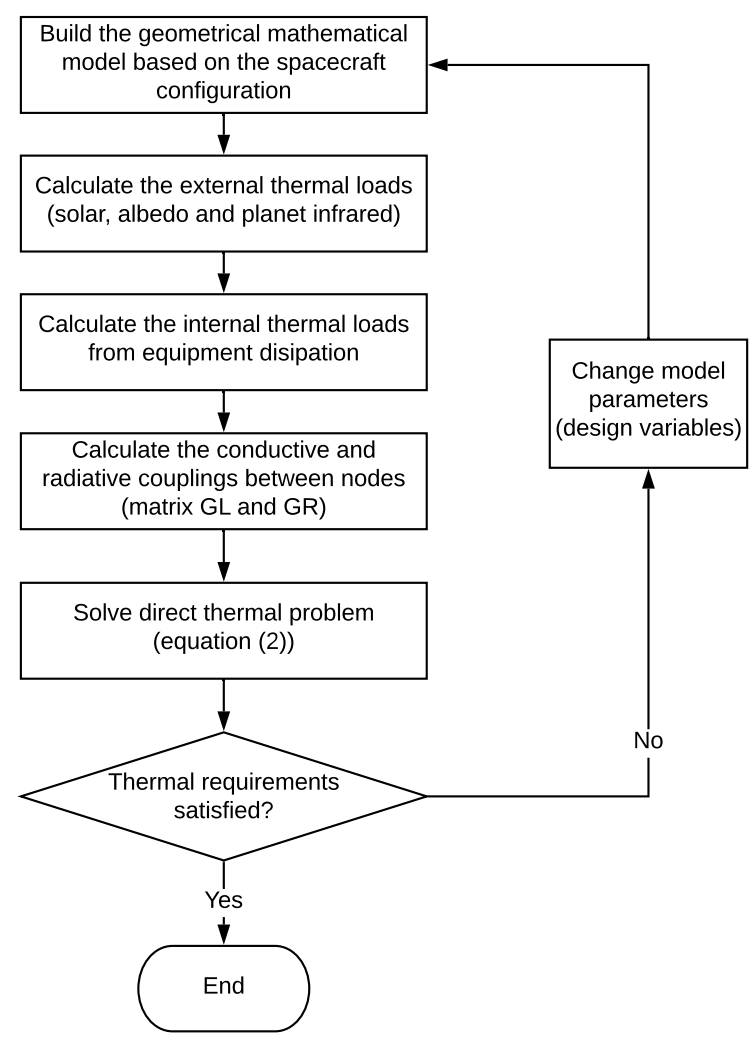

Figure 1. Spacecraft thermal design process

$$
\begin{aligned}
& \text { minimize } f(x, y(x, y)) \\
& \text { with respect to } x \\
& \text { subject to } c_{0}(x, y(x, y)) \leq 0 \\
& c_{i}\left(x_{0}, x_{i}, y_{i}\left(x_{0}, x_{i}, y_{j \neq i}\right)\right) \leq 0 \text { for } i=1 \ldots N,
\end{aligned}
$$

where $f$ is the objective function, $c$ is the constraint function, $x$ are design variables, $y$ are state variables (e.g. temperature), index $i$ means local variables or constraints that affect only one discipline of a total of $N$ disciplines, and index 0 means shared variables or constraints that affect more than one discipline. The objective function can be of any sort of scalar function related to a specific satellite mission, e.g. minimizing the power consumption or maximizing the data rate for communication links. The constraint function for the thermal discipline is certainly the upper and lower bounds of the component's operating and/or survival temperature. The MDF architecture simply means that with each optimizer iteration, the system has to be solved as a whole to return a consistent set of coupling variables. We choose MDF architecture here because it always satisfies the consistency constraints and is easiest to understand from an engineering point of view. The reader is referred to the paper by Martins and Lambe (2013) for more details on multidisciplinary optimization architectures.

Thus, the general MDF formulation for the thermal problem could look like this:

$$
\begin{aligned}
\text { minimize } & f(X) \\
\text { with respect to } & X=\left\{\epsilon, \alpha, k, P_{\text {out }}, \Phi, \Psi, \Omega\right\} \\
\text { subject to } & T_{i, l b}<T_{i}<T_{i, u b} \\
& \sum P_{\text {el }}-\sum Q_{\text {dis }}-\sum P_{\text {out }}=0,
\end{aligned}
$$

where the total vector of design variables $X$ consists of vectors of nodes' emissivity $\epsilon$; absorptivity $\alpha$; thermal conductivity $k$; components' power output $P_{\text {out }}$; and satellite attitude Euler angles $\Phi, \Psi$, and $\Omega$ such that for each node $i$ :

$$
\begin{aligned}
& 0<\epsilon_{\mathrm{i}}<1 \\
& 0<\alpha_{i}<1 \\
& k_{i, l b}<k_{i}<k_{i, u b} \\
& P_{\text {out }_{i, l b}}<P_{\text {out }_{i}}<P_{\text {out }_{i, u b}} \\
& 0<\Phi, \Psi, \Omega<2 \pi .
\end{aligned}
$$

The last equation in (7) defines the equality constraint for conservation of energy where total input electrical power from solar arrays $P_{\mathrm{el}}$ has to equal the sum of the dissipated heat and output power of the sub-systems. The output power is usually in the form of mechanical or electromagnetic energy, which is used to power up electronic instruments and actuators or charge batteries. The overall structure of the multidisciplinary thermal problem and data connections between disciplines are displayed in 
the form of XDSM (Lambe \& Martins, 2012) in Figure 2. The data flow in the Figure 2 is shown as thick gray lines. Components take data inputs from the vertical direction and output data in the horizontal direction. The thin black lines show the process flow, with numbers showing the order in which the components are executed. The thermal optimization problem is composed of three disciplines, which are shown as block components in Figure 2:

1) explicit external heat-flux function;

2) explicit power function;

3) implicit temperature function.

The heat-flux function calculates the absorbed heat flux from external sources, i.e. the Sun, albedo, and planetary infrared, which depend on satellite attitude angles $\Phi, \Psi$, and $\Omega$. The power discipline takes the absorbed fluxes and component power outputs as inputs and calculates internally dissipated heat flux $Q_{\text {dis }}$ and generated electrical power from solar arrays $P_{\mathrm{el}}$. Finally, the temperature discipline assembles matrices $\boldsymbol{G L}$ (eq. 3) and $\boldsymbol{G} \boldsymbol{R}$ (eq. 4) and solves the direct problem (eq. 2) as a system of nonlinear equations. The functions block calculates the objective and constraints, which are fed to the optimizer. The solver is required to converge the residuals of equation (2) to zero and to iteratively solve the multidisciplinary coupled problem (solver loop from steps 1 to 5 ).

Generally, all disciplines could be coupled through temperature-dependent properties, e.g. solar absorptivity, and therefore the external heat flux might depend on temperature. Similarly, solar cell efficiency and thus generated electrical energy also depend on temperature. As shown in Figure 2, the coupling temperature variable is output from the temperature discipline and fed backwards as an input to the power and heat-flux disciplines, thus forming a cycle.

\section{Obtaining the total derivatives of the coupled thermal problem}

To solve the constrained thermal optimization problem defined in section 2 using a deterministic algorithm, the total derivatives of the objective and constraints with respect to the design variables are required. Following

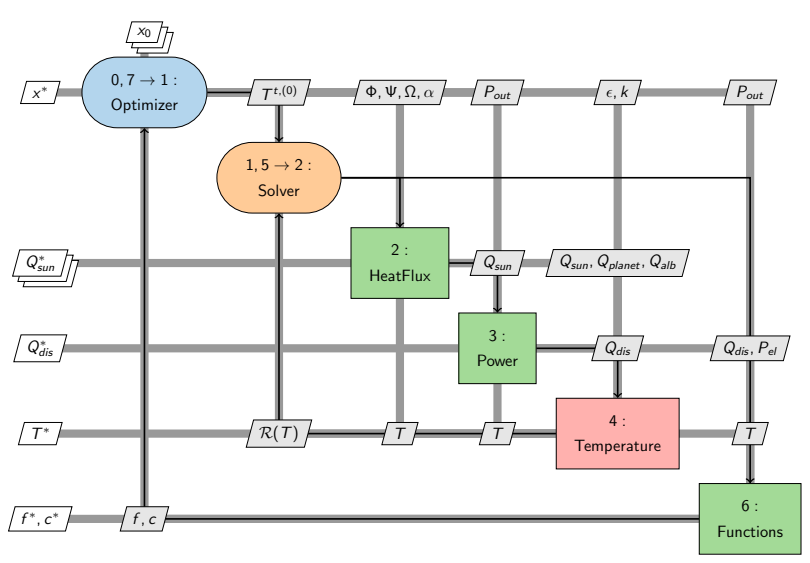

Figure 2. MDF architecture of thermal problem a convention from Gray et al. (2019), we note that total derivatives are defined as the derivatives of the model outputs with respect to the model inputs, while the derivatives of the specific discipline outputs with respect to its inputs are considered to be partial derivatives. As mentioned previously, using finite differences to approximate total derivatives is both inaccurate and computationally inefficient, especially in large-scale models. Obtaining the total analytic derivatives of a coupled multidisciplinary model might seem a non-trivial problem, especially if the model contains implicit functions, such as in our case. Fortunately, there has been a lot of progress recently in deriving efficient methods for calculating analytic total derivatives (e.g. Martins \& Hwang, 2013). In this work, we adopt the MAUD architecture, which was developed by Hwang and Martins (2018) and practically implemented in an open-source multidisciplinary analysis framework called “Open-MDAO” (Gray et al., 2019).

Following the problem formulation in (6), the derivative of the objective or constraint with respect to design variable $x$ is:

$$
\frac{d f}{d x}=\frac{\partial F}{\partial x}+\frac{\partial F}{\partial y} \frac{d y}{d x},
$$

where $f$ (which is a quantity of interest) is distinguished from implicit function $F$, which computes $f$. The key to solving this equation is to obtain the only non-partial derivative $\frac{d y}{d x}$, which captures the change in the converged values of $y$ with respect to $x$. Observing the implicit dependence by $R(x, y)=0$, one obtains a linear system to solve $\frac{d y}{d x}$ :

$$
\frac{\partial R}{\partial y} \frac{d y}{d x}=-\frac{\partial R}{\partial x} .
$$

This is called a direct method. In addition, by substituting $\frac{d y}{d x}$ from equation (10) to equation (9), one obtains the adjoint version:

$$
\frac{d f}{d x}=\frac{\partial F}{\partial x}-\frac{\partial F}{\partial y} \frac{\partial R^{-1}}{\partial y} \frac{\partial R}{\partial x},
$$

where the so-called adjoint vector $\Psi$ is found again by solving a linear system of equations:

$$
\frac{\partial R^{T}}{\partial y} \Psi=\frac{\partial F^{T}}{\partial y}
$$

The direct method requires solving equation (9) $n$ times, where $n$ is the number of variables, whereas the adjoint method is proportional to the number of outputs and independent of the number of inputs. The latter feature is advantageous for large models with a high number of design variables. MAUD provides the mathematical and algorithmic framework that automatically computes the coupled derivatives of the multidisciplinary system via a generalized form of the direct or adjoint method. The total derivatives are computed in a two-step process: 
1) compute the partial derivatives of each component, and

2) solve a linear system of equations that computes the total derivatives (9) or (11).

Therefore, by leveraging the above mentioned technique, we can obtain the total derivatives of the thermal problem by providing just the partial derivatives of each discipline. Here, we provide the exact derivatives of the matrix equation (2) with respect to all of it variables:

$$
\begin{aligned}
& \frac{\partial R}{\partial T}=\boldsymbol{G L}+4 \cdot \boldsymbol{G R} \cdot \operatorname{diag}\left(\{T\}^{3}\right) ; \\
& \frac{\partial R}{\partial G L}=\{T\}^{\top} \otimes \boldsymbol{I} ; \\
& \frac{\partial R}{\partial G R}=\left(\{T\}^{4}\right)^{\top} \otimes \boldsymbol{I} ; \\
& \frac{\partial f}{\partial Q}=I ;
\end{aligned}
$$

where we refer to the residual function of equation (2) as $R$. The partial derivatives of the other disciplines are application specific and examples will be given in the next section.

\section{Practical application of gradient-based spacecraft thermal model optimization}

\subsection{Description of a thermal model}

To demonstrate the use of the technique described in section 3, we present an example spacecraft shown in Figure 3(a).

It is a nano-spacecraft that closely resembles a socalled CubeSat standard (Heidt et al., 2000). The spacecraft consists of a cube-shaped body with a side length of $88 \mathrm{~mm}$ and three solar arrays. One of the arrays is fixed to the body, while the remaining two arrays are deployed after the spacecraft is launched into space. The mission objective is to design this spacecraft for a very wide range of thermal conditions. Therefore, we assume that it must survive the thermal environment expected during an interplanetary mission orbit with an aphelion at $3 \mathrm{AU}$, perihelion at $1 \mathrm{AU}$, and zero inclination. As the solar heat flux follows the square law of distance, spacecraft receive nine times less heat from the Sun at 3 AU compared to conditions in low Earth orbit. The blue rectangles in Figure 3(b) represent spacecraft sub-systems. The requirement for thermal design is to keep sub-system temperatures within an allowable range (shown in Table 1), while maximizing sub-system 1 power allocation during the mission.

A simple lumped-parameter representation of this spacecraft's thermal model is shown in Figure 3(b). The model is cut through the YZ plane to show the internal view, and different colors show the surface optical properties of different parts of the geometry. The initial optical properties are listed in Table 2. The model is discretized into rectangular shells, with each shell representing an individual thermal node. The node numbers of each shell are shown in Figure 3(b). Each deployable solar array consists of two nodes representing the front (nodes 7,8 ) and back (nodes 6,9 ) surfaces of the panel. The nodes or subvolumes are assumed to be isothermal, and physical properties are assumed to be constant within a node. The body of the spacecraft is made of aluminum, while solar arrays and sub-systems are composed of PCBs. For steady-case thermal analysis, the thermal conductivity of the materials needs to be defined. We set the thermal conductivity of aluminum and PCB to 130 and $20 \mathrm{Wm}^{-1} \mathrm{~K}^{-1}$, respectively. The remaining linear conductors connecting different nodes are shown in Figure 3 as jagged lines. Conductors 4-10 and 1-15 represent mechanical spacers through which PCBs are attached to structure. Conductors 5-11,

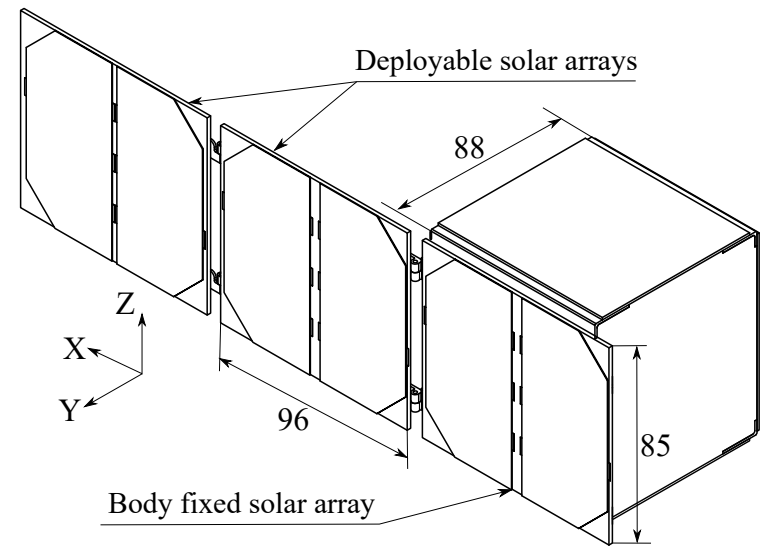

(a)

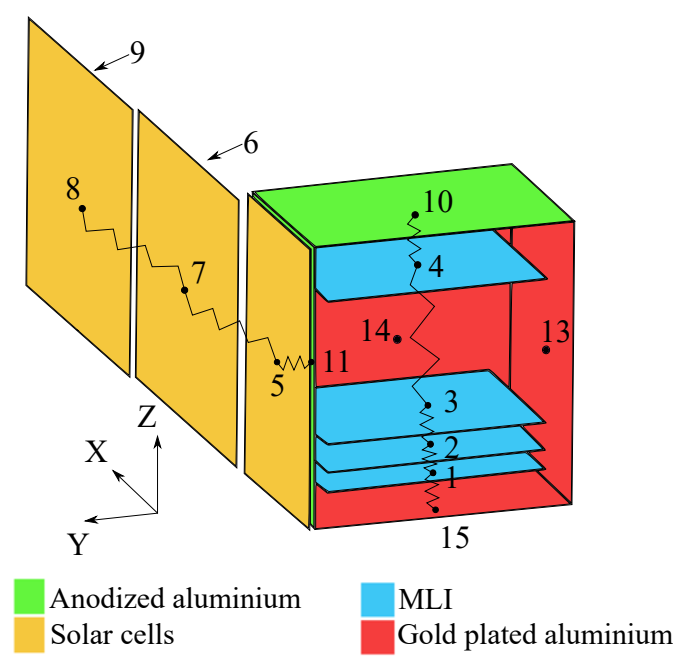

(b)

Figure 3. Spacecraft geometry (a) and thermal model (b). Jagged lines in (b) show linear conductors between thermal nodes. Back surfaces of deployable solar arrays (nodes 6 and 9) are covered with MLI 
Table 1. Thermal requirements of the sub-systems (source: author, 2020)

\begin{tabular}{|c|c|c|}
\hline $\begin{array}{c}\text { Sub-system } \\
\text { number }\end{array}$ & $\begin{array}{c}\text { Minimum } \\
\text { temperature }\end{array}$ & $\begin{array}{c}\text { Maximum } \\
\text { temperature }{ }^{\circ} \mathrm{C}\end{array}$ \\
\hline 1 & -10 & +80 \\
\hline 2 & -40 & +85 \\
\hline 3 & -40 & +85 \\
\hline 4 & 0.0 & +45 \\
\hline
\end{tabular}

Table 2. Surface optical properties

\begin{tabular}{|l|c|c|}
\hline \multicolumn{1}{|c|}{ Surface finish } & $\begin{array}{c}\text { Solar } \\
\text { absorptivity }\end{array}$ & $\begin{array}{c}\text { Infrared } \\
\text { emissivity }\end{array}$ \\
\hline Solar cells & 0.91 & 0.89 \\
\hline MLI & 0.035 & 0.14 \\
\hline Gold-plated aluminum & 0.02 & 0.19 \\
\hline Anodized aluminum & 0.11 & 0.50 \\
\hline
\end{tabular}

5-7, and 7-8 represent solar array hinges. We assume that the sub-systems are well insulated with MLI and that the internal body surface is covered with a low emissivity finish so that the dominant heat path from/to the subsystems is through conduction instead of radiation. In this model, the sides of the body cube are assumed to be fused together without any thermal contact resistance, but the conductors are not shown in Figure 3(b) for the purpose of clarity.

\subsection{Discipline models}

As explained in section 2, the multidisciplinary thermal problem consists of heat-flux, power, and temperature disciplines. We apply the same topology to the example problem presented here, with the distinction that only power and temperature will be coupled. The heat-flux discipline calculates the incident solar radiation flux absorbed by a satellite surface, given by:

$$
Q_{\text {Sun }}=q_{s} \cdot A \cdot \alpha,
$$

where $A$ is surface area and $q_{s}$ is incident solar flux density, calculated by:

$$
q_{s}=G_{s} \cdot \cos (\phi) \cdot d^{-2},
$$

where $d$ is the distance from the Sun in AU, $\phi$ is the angle between incident Sun ray and surface area normal, and the average value of the solar constant $G_{s}$ at one AU distance is $1367 \frac{W}{m^{2}}$ (Gilmore, 2002). Note that the angle $\phi$ needs to be determined for every external surface of the spacecraft and will depend on the design variable $\Phi$. The dependence of these variables might be determined analytically if the model geometry is simple, but in general, a graphical ray-tracing or rendering algorithm is used for more complex geometries to calculate $q_{\mathrm{s}}$ directly. In case a ray-tracing algorithm is used, however, the resulting out- put is not a continuous function, but an array of outputs at specified conditions. To smooth out the data, some kind of surrogate model is typically used, e.g. B-spline interpolant (Hwang et al., 2014).

The power discipline takes the absorbed fluxes and component power outputs as inputs and calculates the internally dissipated heat flux $Q_{d i s}$ and generated electrical power from solar arrays $P_{e l}$. First, the electrical power is calculated as

$$
P_{e l}=Q_{S u n} \cdot \frac{\eta}{\alpha_{s c}},
$$

where $\eta$ is the overall input efficiency given by the product of electrical cell efficiency $\eta_{e}$ and MPPT converter efficiency $\eta_{\text {mppt }}$ :

$$
\eta=\eta_{e} \cdot \eta_{m p p t},
$$

and $\alpha_{s c}-$ is the solar absorptivity of the solar cell. We note that $\eta_{e}$ depends on temperature, which is explained later. The dissipated heat in the solar arrays is then given:

$$
Q_{d i s, s a}=Q_{s u n}-P_{e l}=Q_{S u n} \cdot\left(1-\frac{\eta}{\alpha_{s c}}\right) .
$$

For internal sub-systems, we just assume that all electrical power is converted as heat:

$$
Q_{d i s_{\text {int }}}=P_{\text {out }} \text {, }
$$

Although in general cases, this depends on the nature of the specific sub-system component. Finally, the temperature discipline assembles matrices $\boldsymbol{G L}$ (eq. 3) and $\boldsymbol{G R}$ (eq. 4) and solves the system of nonlinear equations to obtain the temperature of each thermal node. For radiative conductor GR, two cases can be distinguished:

1. Thermal radiation exchange of external surfaces with a deep space environment.

2. Thermal radiation exchange between surfaces inside the spacecraft.

In case of external radiation, equation (4) simplifies to:

$$
G R_{i j}=\sigma \cdot \epsilon_{i} \cdot A_{i} \cdot F_{i j} \text {. }
$$

Unfortunately, for internal radiation, multiple reflections need to be accounted for to achieve accurate results of radiative conductors. This requires GR values to be recalculated for every surface in a spacecraft enclosure whenever emissivity changes. Since radiative solvers rely on Monte Carlo ray-tracing algorithms for view factor calculation, gradient-based optimization of internal surface emissivity becomes problematic. Practically, this is not an issue, however, because external radiation has a dominant effect on thermal equilibrium. Only optimization of external surface optical properties is considered in this paper.

As we need to solve the thermal model for two different boundary conditions, equation (2) has to be modified to obtain a solution at multiple design points:

$$
G L \cdot T+G R \cdot T^{4}+Q=0,
$$

where $T$ and $Q$ are matrices when each column represents a single design point. The size of $T$ and $Q$ is $n \times m$, where 


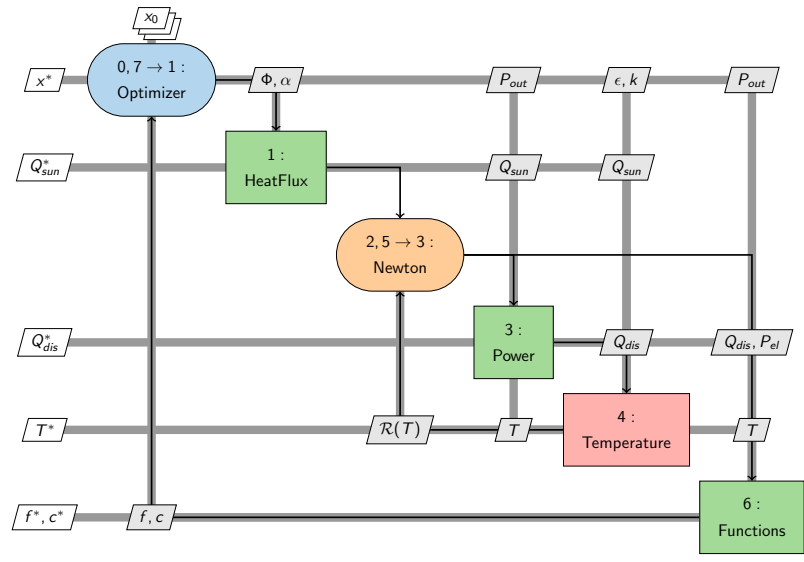

Figure 4. MDF architecture of example thermal problem

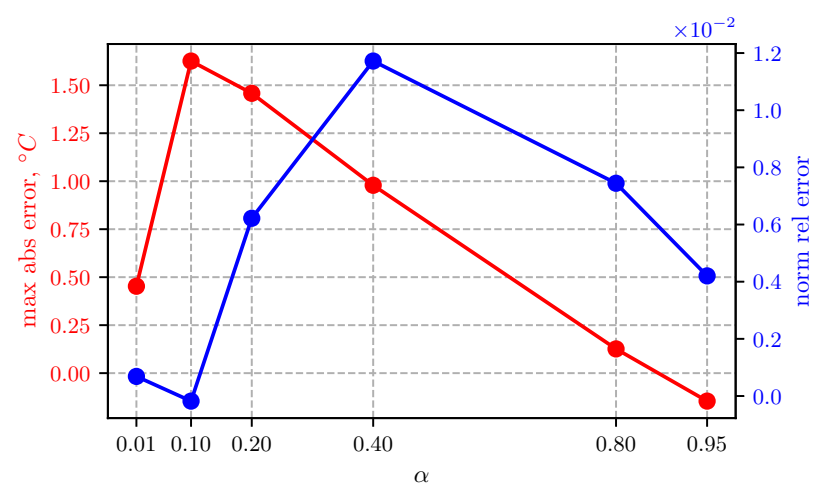

Figure 5. Evolution of maximum absolute (red) and normalized relative (blue) errors for varying infrared emissivity of CubeSat surfaces 6, 9, and 14 $n$ is the number of thermal nodes and $m$ is the number of design points. In our example problem, $n=15$ and $m=2$. Note that $\boldsymbol{G L}$ and $\mathbf{G R}$ are matrices of size $n \times n$.

As mentioned previously, the temperature and power disciplines are coupled because the electrical efficiency of a solar cell depends on temperature. This dependency can be estimated by either using an electric solar cell model or by approximating experimental data. Here, we follow a second approach by applying a linear approximation to the experimental data of AZUR SPACE triple-junction solar cells, as provided in Baur et al. (2017). We assume that the heat-flux discipline is not coupled and can be executed sequentially with no iteration.

The overall MDF architecture of the example problem is shown in Figure 4. Here, we use a nonlinear iterative Newton solver to converge the coupled power and temperature discipline group by driving the residuals of the nonlinear system of equations to zero. The multidisciplinary thermal model was implemented in an open-source multidisciplinary analysis framework called OpenMDAO (Gray et al., 2019), which focuses on supporting gradientbased optimization with analytic derivatives.

\subsection{Evaluation of thermal solver accuracy}

The assumption in this analysis is that external radiation can be modeled with equation (23), i.e. there are no reflections of infrared radiation. For the considered example with deployable solar arrays, this assumption is of course no longer true due to possible reflections from deployable array back surfaces. To test the effect of reflections and the overall accuracy of the thermal solver, the model was run for varying infrared emissivity of CubeSat surfaces 6, 9, and 14. Other thermal properties were kept constant. The results were compared with those obtained for the same model in the Esatan-TMS commercial thermal analysis program, which is a standard spacecraft thermal analysis tool used in the European Space Agency. Results are summarized in Figure 5. The maximum measured absolute error is $1.63^{\circ} \mathrm{C}$ at surface emissivity of 0.10 . The error is positive as expected because in reality, additional re- flections cause an increase in the total radiation exchange factor from associated geometry to deep space. This causes larger temperatures to be reported in OpenMDAO than there actually is. As the emissivity is decreased to lower values, the effect diminishes because of relatively lower heat flux compared to other satellite surfaces. As expected, increasing surface emissivity decreases the errors because in this case, the radiation exchange factor approaches the value given by equation (23).

\subsection{Set-up of the optimization problem}

The optimization objective is to maximize the power allocation to sub-system 1 by changing the values of the optical properties of anodized aluminum, thermal conductance between sub-systems and structure, thermal conductance of solar panels hinges, the power allocation to sub-system 4 , and the attitude yaw angle $\Phi$ around spacecraft body axis $Z$. The angle $\Phi$ changes the orientation of the spacecraft with respect to the Sun, where 0 angle corresponds to the Sun vector coincident with the $\mathrm{Y}$ body axis. The inequality constraints for the problem are the upper and lower temperature bounds listed in Table 1. In addition, an equality constraint for energy conservation has to be complied with. The problem formulation is summarized in Table 3.

The design variables for power allocation and yaw angle as well as constraints need to be parametrized with respect to different design points. As mentioned previously, in this example, we have to solve the problem at two design points: the hot case at $1 \mathrm{AU}$ distance from the Sun and the cold case at $3 \mathrm{AU}$ distance from the Sun. Emissivity is optimized for the whole external surface because its effect does not depend on satellite attitude. The absorptivity for node 11 is not optimized as this side is shadowed by a body solar array. To comply with the equality constraint, we allow the optimizer to freely choose the amount of electrical power allocated to sub-systems 1 and 4 and set the remaining unused electrical power term as dissipated heat in the structure. This feature is required to limit the heat dissipation in sub-systems if they get too hot. 
Table 3. Example optimization problem

\begin{tabular}{|c|c|c|c|c|}
\hline \multicolumn{2}{|c|}{ Variable/function } & Units & Description & Quantity \\
\hline maximize & $\sum_{i=1}^{2} P_{\text {out }, 1_{i}}$ & $\mathrm{~W}$ & The sum power of sub-system 1 at two design points & 1 \\
\hline \multirow[t]{7}{*}{ with respect to } & $0<\epsilon<1$ & - & Emissivity of external body surface (nodes 10-15) & 1 \\
\hline & $0<\alpha_{12}<1$ & - & Absorptivity of body side X- (node 12) & 1 \\
\hline & $0.25<k_{\text {spacer }}<237$ & $W m^{-1} K^{-1}$ & Conductivity of spacers connecting nodes $4-10,1-15$ & 2 \\
\hline & $0.004<k_{\text {hinge }}<0.1$ & $W K^{-1}$ & $\begin{array}{l}\text { Conductance of hinges connecting nodes } 7-8,5-7 \text {, and } \\
5-11\end{array}$ & 3 \\
\hline & $0.25<P_{\text {out }}<7$ & $\mathrm{~W}$ & Power allocation to sub-systems 1 and 5 & $2 \times 2$ \\
\hline & $0.25<Q_{d i s}<7$ & $\mathrm{~W}$ & Unused power dissipated as heat on body structure & $1 \times 2$ \\
\hline & $0<\Phi<\frac{\pi}{2}$ & $\mathrm{rad}$ & Yaw attitude angle with respect to Sun vector & $1 \times 2$ \\
\hline \multicolumn{4}{|r|}{ Total number of design variables } & 15 \\
\hline \multirow[t]{2}{*}{ subject to } & $T_{i, l b}<T_{i}<T_{i, u b}$ & & Temperature constraints from Table 1 & $4 \times 2$ \\
\hline & $\sum P_{e l}-\sum Q_{d i s}-\sum P_{o u t}=0$ & & Conservation of energy constraint & $1 \times 2$ \\
\hline \multicolumn{4}{|c|}{ Total number of constraints } & 10 \\
\hline
\end{tabular}

The SLSQP was selected as the gradient-based optimization algorithm as it can minimize a function of several variables with any combination of bounds, equality, and inequality constraints. OpenMDAO implementation of SLSQP was originally implemented by Kraft (1988).

\subsection{Optimization results}

First, the performance of the analytic total derivative computation was tested on varying thermal-model mesh density by increasing the number of nodes from 15 to 73 and 225 (Figure 6). As can be seen from Figure 7(a), the computation time of total derivatives increases almost linearly with mesh density. As suggested by theory, however, the computation time does not depend on the number of design variables, as seen in Figure 7(b). Increasing mesh density also affects the accuracy of the solution, e.g. the relative change of temperature of sub-system 1 is $-9 \%$ (73 nodes) and $-4 \%$ (225 nodes) with successive mesh refinement.

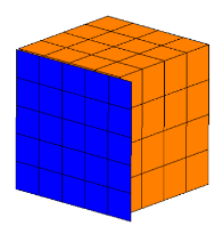

$n=225$

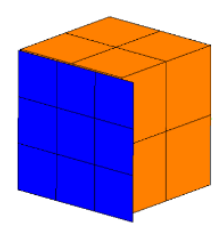

$n=73$

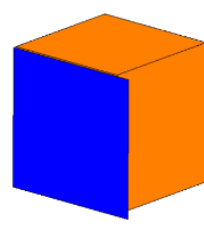

$n=15$
Figure 6. Varying fidelity of thermal model mesh, where $n$ is the total number of thermal nodes. Deployable solar arrays are meshed identically as fixed array (blue), but are not shown for clarity
To avoid sub-optimal solutions when the optimizer gets trapped in local extremum, the model was run starting with different initial values. The baseline initial solution was obtained starting with the initial values listed in Table 4; after that, the LHS method was used to obtain equally distributed samples of starting input variable values. The model with 73 thermal nodes in Figure 6 was used. The number of samples was increased until no improvement in objective function value was observable. The optimized design variable values for the initial local and global solutions are shown in Table 4 and corresponding temperatures are shown in Figure 8. As can be seen from Figure 9, the best solution is obtained when the number of initial variable samples is increased to 30 . This could be expected from a gradient-based optimizer and suggests that the designer should be aware of this limitation.

It is evident from the results in Table 4, however, that even the local solution provides a hint to the designer about the direction of improvement. Mainly, by increasing the angle $\Phi$ during the hot case and decreasing the absorptivity of body surface, the satellite absorbs less heat from the Sun and more power can be dissipated in subsystem 1 without it overheating, even though the total electrical power input is reduced. The evolution of objective and constraint violation values for local and global solution optimizer runs are shown in the Figure 10. Constraints are aggregated for both design points, positive values indicate that the constraint is violated. Temperature 


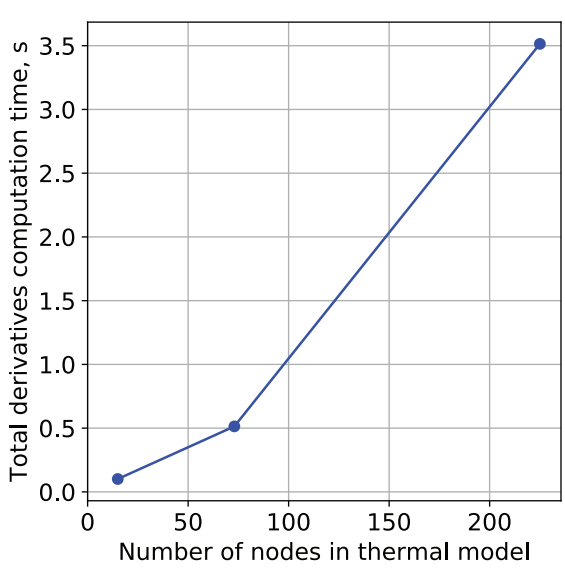

(a)

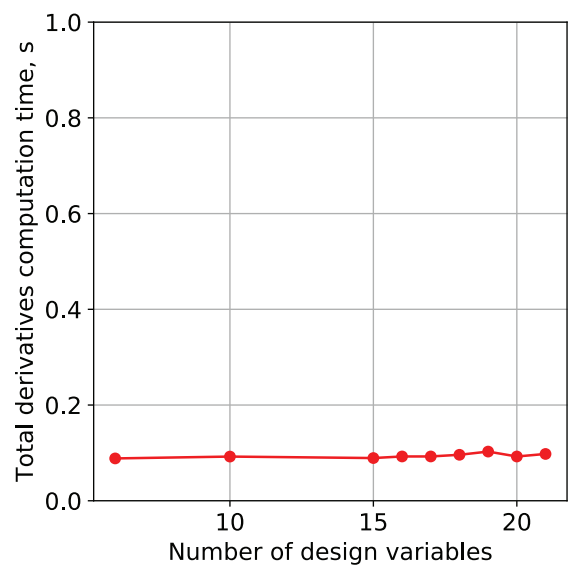

(b)

Figure 7. Time for computing total derivatives of objective and constraints with respect to design variables for (a) varying model fidelity and (b) varying number of design variables (platform: Intel Core i5-5300U)

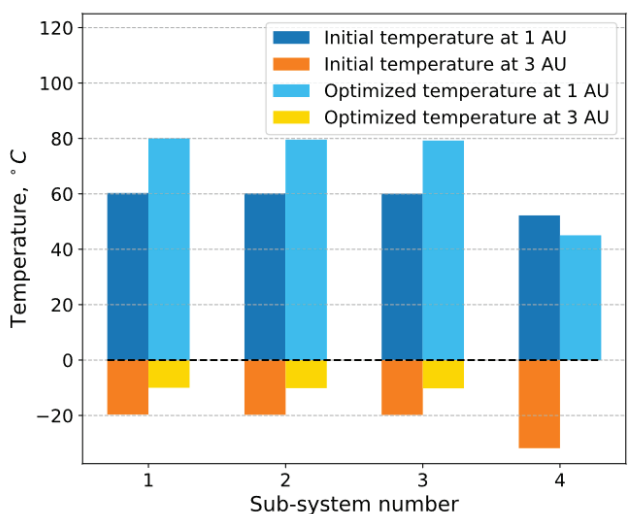

Figure 8. Initial and optimized temperatures. Sub-system 4 temperature at $3 \mathrm{AU}$ after optimization is zero indices indicate sub-system numbers, $l b$ - lower bound, up - upper bound. Only 10 function evaluations were required to achieve a local extremum during the initial run (Figure 10a), whereas approximately 50 were required for a global solution (Figure 10b). By comparing Figures 10a and $10 \mathrm{~b}$, one can see that although the initial violation of constraints for the best run was much higher, it was still possible to achieve constraint feasibility. In fact, from a total of 100 optimization runs, the constraint feasibility was not reached only once within a given iteration limit of 70 iterations. This suggests that the gradient-based optimizer and suggested MDF architecture are robust in terms of nearly always providing the designer with a feasible solution.

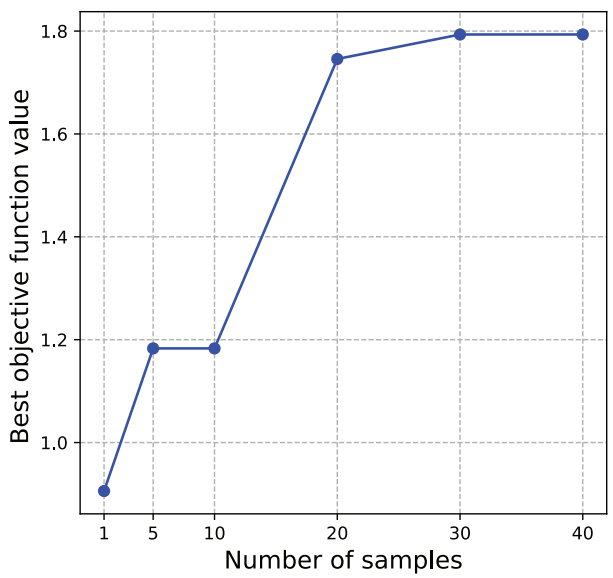

(a)

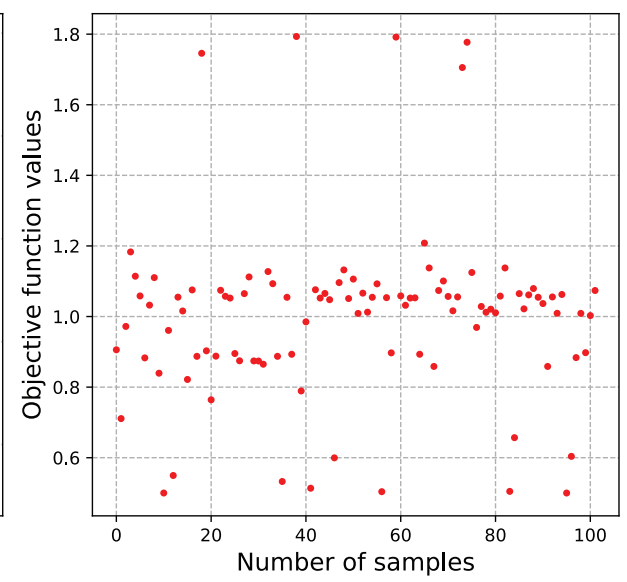

(b)

Figure 9. Evolution of best objective value with (a) number of samples and (b) plot of all objective results 
Table 4. Initial and optimized values of design variables (vector variables shown in brackets)

\begin{tabular}{|c|c|c|c|}
\hline \multirow{2}{*}{$\begin{array}{c}\text { Design } \\
\text { variable }\end{array}$} & \multirow{2}{*}{ Initial values } & \multicolumn{2}{|c|}{ Optimized values } \\
\hline & & $\begin{array}{c}\text { Initial } \\
\text { optimizer run }\end{array}$ & $\begin{array}{l}\text { Best solution } \\
\text { optimizer run }\end{array}$ \\
\hline$\epsilon$ & 0.11 & 0.94 & 0.71 \\
\hline$\alpha_{12}$ & 0.5 & 0.07 & 0.07 \\
\hline$k_{\text {spacer }, 4-10}$ & 6.7 & 2.88 & 6.33 \\
\hline$k_{\text {spacer }, 1-5}$ & 6.7 & 2.96 & 1.83 \\
\hline$k_{\text {hinge, } 7-8}$ & 0.02 & 0.1 & 0.1 \\
\hline$k_{\text {hinge, } 5-7}$ & 0.02 & 0.1 & 0.1 \\
\hline$k_{\text {hinge,5-11 }}$ & 0.02 & 0.004 & 0.1 \\
\hline$P_{\text {out }, 1}$ & {$[0.3,0.3]$} & {$[0.51,0.4]$} & {$[1.17,0.62]$} \\
\hline$P_{\text {out }, 4}$ & {$[0.25,0.25]$} & {$[0.25,0.46]$} & {$[0.25,0.25]$} \\
\hline$P_{d i s}$ & {$[0,0]$} & {$[5.75,0]$} & {$[0.25,0]$} \\
\hline$\Phi$ & {$[0,0]$} & {$[10.5,0]$} & {$[77.2,0]$} \\
\hline Objective $\mathrm{f}$ & tion value & 0.91 & 1.79 \\
\hline
\end{tabular}
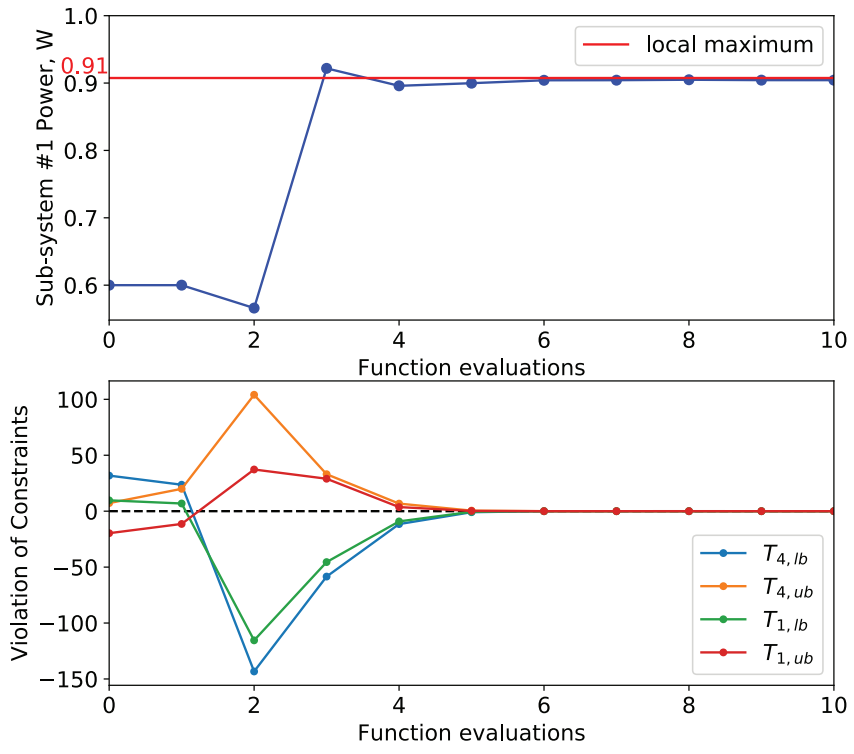

(a)

\section{Conclusions}

In this paper, we have presented MDF architecture for the multidisciplinary analysis and optimization of spacecraft thermal design. The architecture consists of three heterogenous computational models: external heat-flux, sub-system power allocation, and steady-case heat transfer. The multidisciplinary formulation of the problem facilitates the integration of thermal design into the overall spacecraft design process by capturing the dependency of the thermal model on other disciplines. We then showed how to derive analytic total derivatives of the coupled computational model using the adjoint method. It was found that the model results agreed well with those obtained in Esatan-TMS thermal analysis software. The worst case absolute and relative errors for a CubeSat model with solar arrays were below $1.63{ }^{\circ} \mathrm{C}$ and $1.2 \%$, respectively. The cause of the error can be mainly attributed to the reflections from deployable geometry that are not accounted for in the integrated OpenMDAO model. By using this method together with the SLSQP optimization algorithm, we solved a small interplanetary spacecraft thermal optimization problem consisting of one objective, 15 design variables, and 10 constraints. Newton's method was used to converge the coupled nonlinear steady-case heat transfer and power discipline models.

The optimization routine was repeated for a varying number of design space samples obtained using the LHS method. The best objective function solution was obtained
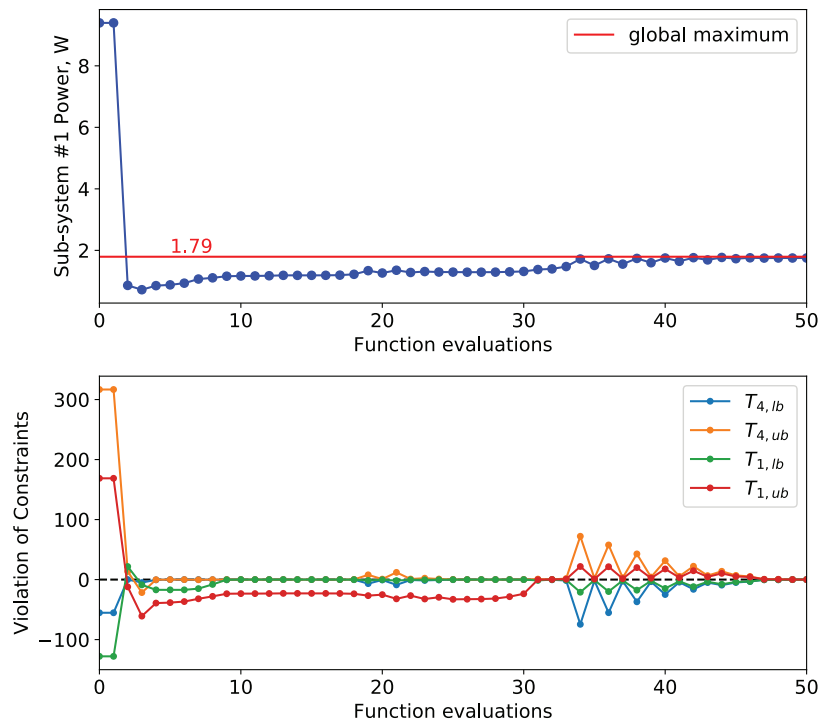

(b)

Figure 10. Optimization progress of objective function and temperature constraint (in Kelvin scale) violations for (a) the initial optimizer run and (b) the best solution optimizer run. 
when the number of initial variable samples was increased to 30 , which resulted in $100 \%$ improvement compared to the initial solution. No improvement was observed by increasing the number of samples above 30 . Due to the efficient computation of total derivatives, all 30 samples were run and the best solution was obtained in less than half an hour on an Intel Core i5-5300U processor for a lumped-parameter thermal model consisting of 73 nodes. It was noted that even given a sub-optimal solution, the optimization results provided a hint to the designer about the direction of improvement. We think this feature of MDF solution architecture and gradient-based optimization is beneficial because in nearly every case, the optimizer returns a feasible solution regardless of the outcome of the optimization. In fact, we observed that from a total of 100 optimization runs, the constraint feasibility was not reached only once within a given iteration limit of 70 iterations.

We further demonstrated that the adjoint method can compute analytic total derivatives of the computational model regardless of the number of design variables, which is relevant for models containing many design variables. The cost of computing total derivatives does depend on the size of the problem though. In our case, by varying thermal model mesh density from 15 to 225 lumped-parameter thermal nodes, we observed almost a linear increase in derivative computation time from $0.1 \mathrm{~s}$ to $3.5 \mathrm{~s}$. We believe that the gradient-based optimization of multidisciplinary thermal models presented in this research work could find a practical application in spacecraft or similar aerospace system design tasks containing large numbers of design variables and conflicting thermal requirements.

\section{Disclosure statement}

The authors confirm that they do not have any competing financial, professional, or personal interests from other parties.

\section{References}

Baur, C., Khorenko, V., Siefer, G., Inguimbert, V., Park, S., Boizot, B., Bourgoin, Jacques C., Casale, M., Campesato, R., Schnell, H., Gerhard, A., Zanella, P., Ferrando, E., Reutenauer, X., Bongers, E., \& Gras, A. (2017). Status of solar generator related technology development activities supporting the juice mission. E3S Web of Conferences, 16, 04005. https://doi.org/10.1051/e3sconf/20171604005

Bodie, M., Russell, G., McCarthy, K., Lucus, E., Zumberge, J., \& Wolff, M. (2010). Thermal analysis of an integrated aircraft model. Paper presented at the 48th AIAA Aerospace Sciences Meeting Including the New Horizons Forum and Aerospace Exposition. Orlando, Florida. https://doi.org/10.2514/6.2010-288

Chari, N. (2009). Spacecraft thermal design optimization (Doctoral dissertation). University of Toronto, Toronto, Canada.

Escobar, E., Diaz, M., \& Zagal, J. C. (2016). Evolutionary design of a satellite thermal control system: Real experiments for a CubeSat mission. Applied Thermal Engineering, 105, 490-500. https://doi.org/10.1016/j.applthermaleng.2016.03.024
Falck, R. D., Chin, J. C., Schnulo, S. L., Burt, J. M., \& Gray, J. S. (2017). Trajectory optimization of electric aircraft subject to subsystem thermal constraints. Paper presented at the 18th AIAA/ISSMO Multidisciplinary Analysis and Optimization Conference. Denver, CO. https://doi.org/10.2514/6.2017-4002

Forrester, A. I. J., Sóbester, A., \& Keane, A. J. (2008). Engineering design via surrogate modelling. Wiley. https://doi.org/10.1002/9780470770801

Galski, R. L., De Sousa, F. L., Ramos, F. M., \& Muraoka, I. (2007). Spacecraft thermal design with the generalized extremal optimization algorithm. In H. R. B. Orlande \& M. J. Colaço (Eds.), Inverse Problems in Science and Engineering, Vol. 15, (pp. 61-75). Taylor \& Francis Group. https://doi.org/10.1080/17415970600573924

Gilmore, D. G. (2002). Spacecraft thermal control handbook (Vol. I). Aerospace Press. https://doi.org/10.2514/4.989117

Gray, J. S., Hwang, J. T., Martins, J. R. R. A., Moore, K. T., \& Naylor, B. A. (2019). OpenMDAO: An open-source framework for multidisciplinary design, analysis, and optimization. Structural and Multidisciplinary Optimization, 59(4), 10751104. https://doi.org/10.1007/s00158-019-02211-Z

Heidt, H., Puig-Suari, J., Moore, A., Nakasuka, S., \& Twiggs, R. (2000). CubeSat: A new generation of picosatellite for education and industry low-cost space experimentation. Paper presented at the 14th Annual AIAA/USU Conference on Small Satellites. Logan, UT. https://digitalcommons.usu.edu/smallsat/2000/All2000/32

Hwang, J. T., Lee, D. Y., Cutler, J. W., \& Martins, J. R. R. A. (2013). Large-scale MDO of a small satellite using a novel framework for the solution of coupled systems and their derivatives. Paper presented at the Proceedings of the 54th AIAA/ ASME/ASCE/AHS/ASC Structures, Structural Dynamics, and Materials Conference. Boston, MA. https://doi.org/10.2514/6.2013-1599

Hwang, J. T., Lee, D. Y., Cutler, J. W., \& Martins, J. R. R. A. (2014). Large-scale multidisciplinary optimization of a small satellite's design and operation. Journal of Spacecraft and Rockets, 51(5), 1648-1663. https://doi.org/10.2514/1.A32751

Hwang, J. T., \& Martins, J. R. R. A. (2018). A computational architecture for coupling heterogeneous numerical models and computing coupled derivatives. ACM Transactions on Mathematical Software, 44(4), 1-39. https://doi.org/10.1145/3182393

Kraft, D. (1988). A software package for sequential quadratic programming. Forschungsbericht-Deutsche Forschungs- Und Versuchsanstalt Fur Luft-Und Raumfahrt. http://degenerateconic. com/wp-content/uploads/2018/03/DFVLR_FB_88_28.pdf

Lambe, A. B., \& Martins, J. R. R. A. (2012). Extensions to the design structure matrix for the description of multidisciplinary design, analysis, and optimization processes. Structural and Multidisciplinary Optimization, 46(2), 273-284. https://doi.org/10.1007/s00158-012-0763-y

Martins, J. R. R. A., \& Hwang, J. T. (2013). Review and unification of methods for computing derivatives of multidisciplinary computational models. AIAA Journal, 51(11), 25822599. https://doi.org/10.2514/1.J052184

Martins, J. R. R. A., \& Lambe, A. B. (2013). Multidisciplinary design optimization: A survey of architectures. AIAA Journal, 51(9), 2049-2075. https://doi.org/10.2514/1.J051895

Meseguer, J., Pérez-Grande, I., \& Sanz-Andrés, A. (2012). Spacecraft thermal control (1st ed.). Woodhead Pub. https://doi.org/10.1533/9780857096081.1

Sobieszczanski-Sobieski, J. (1990). Sensitivity of complex, internally coupled systems. AIAA Journal, 28(1), 153-160.

https://doi.org/10.2514/3.10366 
Stout, K. D., Masterson, R. A., \& Miller, D. W. (2013). Design optimization of thermal paths in spacecraft systems. MIT. http:// ssl.mit.edu/files/website/theses/SM-2013-StoutKevin.pdf

Wang, D., Naterer, G. F., \& Wang, G. (2002). Adaptive response surface method for thermal optimization: Application to aircraft engine cooling system. Paper presented at the 8th AIAA/ ASME Joint Thermophysics and Heat Transfer Conference. St. Louis, MI. https://doi.org/10.2514/6.2002-3000

\section{Notations}

Variables and functions

$A$ - cross-sectional area;

$C$ - heat capacity;

$c$ - constraint function;

$d$ - distance to the Sun;

$F$ - implicit function;

$f$ - objective function;

$F_{i j}$ - the geometric view factor between surface $i$ and $j$;

$G L$ - linear thermal conductance factor;

$G R$ - radiative thermal conductance factor;

$G_{\text {s }}$ - solar constant;

$k$ - thermal conductivity;

$L$ - the distance between two adjacent thermal nodes;

$m$ - number of design points;

$N$ - number of disciplines;

$n$ - number of thermal nodes in the model;

$P_{e l}$ - electric input power;

$P_{\text {out }}$ - component output power;

$Q$ - heat flux;

$Q_{\text {alb }}$ - Albedo heat flux;

$Q_{\text {dis }}$ - internally dissipated heat flux;

$Q_{\text {planet }}$ - planetary infrared heat flux;
$Q_{\text {Sun }}$ - absorbed solar heat flux;

$q_{\mathrm{s}}$ - incident solar flux density;

$R$ - function of residual equilibrium equations;

$T$ - temperature;

$t$ - time;

$X-$ vector of design variables;

$x$ - design variable;

$y$ - state variable;

$\alpha$ - solar absorptivity;

$\eta$ - solar array efficiency;

$\eta_{e}$ - electrical efficiency of a solar cell;

$\eta_{\text {mppt }}$ - efficiency of MPPT converter.

$\sigma$ - Stefan-Boltzmann constant;

$\Phi$ - yaw Euler angle;

$\Psi$ - pith Euler angle;

$\Psi$ - adjoint vector;

$\Omega$ - roll Euler angle;

$\epsilon$ - infrared emissivity;

\section{Abbreviations}

AU - astronomical unit, the average distance between the Earth and the Sun, which is about 93 million miles or 150 million kilometers

LHS - Latin hypercube sampling;

MAUD - modular analysis and unified derivatives architecture;

MDF - multidisciplinary feasible;

MLI - multi-layer insulation;

MPPT - maximum power point tracker;

PCB - printed circuit board;

SLSQP - sequential least squares programming;

XDSM - extended design structure matrix. 